ARTICLE

\title{
Ultra-dense optical data transmission over standard fibre with a single chip source
}

\author{
Bill Corcoran (i) ${ }^{1 凶}$, Mengxi Tan², Xingyuan Xu (i) ${ }^{2}$, Andreas Boes (i) ${ }^{3}$, Jiayang $\mathrm{Wu}^{2}$, Thach G. Nguyen ${ }^{3}$,
} Sai T. Chu (1) ${ }^{4}$, Brent E. Little ${ }^{5}$, Roberto Morandotti (iD ${ }^{6,7}$, Arnan Mitchell (iD ${ }^{3} \&$ David J. Moss (iD) ${ }^{2 \times}$

Micro-combs - optical frequency combs generated by integrated micro-cavity resonators offer the full potential of their bulk counterparts, but in an integrated footprint. They have enabled breakthroughs in many fields including spectroscopy, microwave photonics, frequency synthesis, optical ranging, quantum sources, metrology and ultrahigh capacity data transmission. Here, by using a powerful class of micro-comb called soliton crystals, we achieve ultra-high data transmission over $75 \mathrm{~km}$ of standard optical fibre using a single integrated chip source. We demonstrate a line rate of 44.2 Terabits $\mathrm{s}^{-1}$ using the telecommunications C-band at $1550 \mathrm{~nm}$ with a spectral efficiency of 10.4 bits $\mathrm{s}^{-1} \mathrm{~Hz}^{-1}$. Soliton crystals exhibit robust and stable generation and operation as well as a high intrinsic efficiency that, together with an extremely low soliton micro-comb spacing of $48.9 \mathrm{GHz}$ enable the use of a very high coherent data modulation format (64 QAM - quadrature amplitude modulated). This work demonstrates the capability of optical micro-combs to perform in demanding and practical optical communications networks.

\footnotetext{
${ }^{1}$ Photonic Communications Lab, Department of Electrical and Computer System Engineering, Monash University, Clayton, VIC 3168 , Australia. ${ }^{2}$ Centre for Micro-Photonics, Swinburne University of Technology, Hawthorn, VIC 3122, Australia. ${ }^{3}$ Integrated Photonics and Applications Centre (InPAC), School of Engineering, RMIT University, Melbourne, VIC 3001, Australia. ${ }^{4}$ Department of Physics and Material Science, City University of Hong Kong, Tat Chee Avenue, Hong Kong, China. ${ }^{5}$ Xi'an Institute of Optics and Precision Mechanics Precision Mechanics of CAS, Xi'an, China. ${ }^{6}$ INRS-Énergie, Matériaux et Télécommunications, 1650 Boulevard Lionel-Boulet, Varennes, Québec J3X 1S2, Canada. ${ }^{7}$ Institute of Fundamental and Frontier Sciences, University of

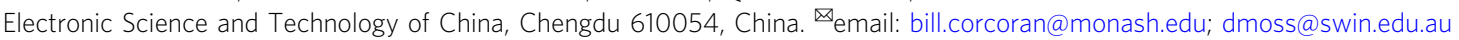


T he global optical fibre network currently carries hundreds of terabits per second every instant, with capacity growing at $\sim 25 \%$ annually ${ }^{1}$. To dramatically increase bandwidth capacity, ultrahigh capacity transmission links employ massively parallel wavelength division multiplexing (WDM) with coherent modulation formats ${ }^{2,3}$, and in recent lab-based research, by using spatial division multiplexing (SDM) over multicore or multimode fibre ${ }^{4}$. At the same time, there is a strong trend towards a greater number of shorter high-capacity links. Whereas core long-haul (spanning 1000's km) communications dominated global networks 10 years ago, now the emphasis has squarely shifted to metro-area networks (linking across 10's-100's km) and even data centres $(<10 \mathrm{~km})$. All of this is driving the need for increasingly compact, low-cost and energy-efficient solutions, with photonic integrated circuits emerging as the most viable approach. The optical source is central to every link, and as such, perhaps has the greatest need for integration. The ability to supply all wavelengths with a single, compact integrated chip, replacing many parallel lasers, will offer the greatest benefits $s^{5,6}$.

Micro-combs, optical frequency combs based on microcavity resonators, have shown significant promise in fulfilling this role ${ }^{7-10}$. They offer the full potential of their bulk counterparts $^{11,12}$, but in an integrated footprint. The discovery of temporal soliton states (DKS-dissipative Kerr solitons) $10,13-17$ as a means of mode-locking micro-combs has enabled breakthroughs in many fields including spectroscopy ${ }^{18,19}$, microwave photonics ${ }^{20}$, frequency synthesis ${ }^{21}$, optical ranging $^{22,23}$, quantum sources 24,25 , metrology 26,27 and more. One of their most-promising applications has been optical fibre communications, where they have enabled massively parallel ultrahigh capacity multiplexed data transmission ${ }^{28-30}$.

The success of micro-combs has been enabled by the ability to phase-lock, or mode-lock, their comb lines. This, in turn, has resulted from exploring novel oscillation states such as temporal soliton states, including feedback-stabilised Kerr combs ${ }^{29}$, dark solitons $s^{30}$ and $\mathrm{DKS}^{28}$. DKS states, in particular, have enabled transmission rates of $30 \mathrm{~Tb} / \mathrm{s}$ for a single device and $55 \mathrm{~Tb} / \mathrm{s}$ by combining two devices, using the full $\mathrm{C}$ and $\mathrm{L}$ telecommunication bands $^{28}$. In particular, for practical systems, achieving a high spectral efficiency is critically important-it is a key parameter as it determines the fundamental limit of data-carrying capacity for a given optical communications bandwidth ${ }^{2,3}$.

Recently ${ }^{17,11}$, a powerful class of micro-comb termed soliton crystals was reported, and devices realised in a CMOS (complementary metal-oxide semiconductor) compatible platform ${ }^{2,3,8,9,31}$ have proven highly successful at forming the basis for microwave and RF photonic devices ${ }^{32,33}$. Soliton crystals were so-named because of their crystal-like profile in the angular domain of tightly packed self-localised pulses within micro-ring resonators (MRRs) ${ }^{17}$. They are naturally formed in micro-cavities with appropriate modecrossings without the need for complex dynamic pumping and stabilisation schemes that are required to generate self-localised DKS waves (described by the Lugiato-Lefever equation ${ }^{34}$ ). The key to their stability lies in their intracavity power that is very close to that of spatiotemporal chaotic states 17,35 . Hence, when emerging from chaotic states there is very little change in intracavity power and thus no thermal detuning or instability, resulting from the 'soliton step' that makes resonant pumping more challenging ${ }^{36}$. It is this combination of intrinsic stability (without the need for external aid), ease of generation and overall efficiency that makes them highly suited for demanding applications such as ultrahigh-capacity transmission beyond a terabit/s.

Here, we report ultrahigh bandwidth optical data transmission across standard fibre with a single integrated chip source. We employ soliton crystals realised in a CMOS-compatible platform $^{31-33}$ to achieve a data line-rate of $44.2 \mathrm{~Tb} / \mathrm{s}$ from a single source, along with a high spectral efficiency of $10.4 \mathrm{bits} / \mathrm{s} / \mathrm{Hz}$. We accomplish these results through the use of a high modulation format of 64 QAM (quadrature amplitude modulation), a low comb-free spectral range (FSR) spacing of $48.9 \mathrm{GHz}$, and by using only the telecommunications C-band. We demonstrate transmission over $75 \mathrm{~km}$ of fibre in the laboratory as well as in a field trial over an installed network in the greater metropolitan area of Melbourne, Australia. Our results stem from the soliton crystal's extremely robust and stable operation/generation as well as its much higher intrinsic efficiency, all of which are enabled by an integrated CMOS-compatible platform.

\section{Results}

Experiment. A schematic illustrating the soliton crystal optical structure is shown in Fig. 1a, with the physical chip shown in Fig. $1 \mathrm{~b}$ and the experimental setup for ultrahigh bandwidth optical transmission in Fig. 1c (also see Methods and Supplementary Note 1). The micro-resonator had an FSR spacing of $48.9 \mathrm{GHz}$ and generated a soliton crystal with this spacing $(\sim 0.4$ $\mathrm{nm}$ ) over a bandwidth of $>80 \mathrm{~nm}$ when pumped with $1.8 \mathrm{~W}$ of continuous-wave (CW) power (in-fibre, incident) at $1550 \mathrm{~nm}$. The soliton crystal micro-comb was generated by automatically tuning the pump laser to a pre-set value. The primary comb and generated soliton crystal states are shown in Figs. $2 a$ and $b$. Figure $2 \mathrm{c}$ demonstrates the stability of the soliton crystal comb generation by showing a variation in individual tone powers of $< \pm 0.9 \mathrm{~dB}$ over 10 different generation instances through wavelength sweeping (from 1550.300 to $1550.527 \mathrm{~nm}$ ). This demonstrates the repeatability of turn-key micro-comb generation from this integrated CMOS-compatible device.

From the generated micro-comb, 80 lines were selected over the telecommunications C-band ( $32 \mathrm{~nm}$ wide, $3.95 \mathrm{THz}$ window from 1536 to $1567 \mathrm{~nm}$ ), which were then flattened with a spectral shaper (WaveShaper $4000 \mathrm{~S}-$ see Methods). Next, the number of wavelengths were effectively doubled to 160 (equivalent to a comb spacing of $24.5 \mathrm{GHz}$ ) to optimise the spectral efficiency (spectrally useful content) by using a single-sideband modulation scheme to generate odd/even de-correlated test channels (see Methods). We then combined a test band of six channels, with the remaining bands providing loading channels having the same odd-and-even channel structure. We used a high order format of 64 QAM to modulate the entire comb at a symbol rate of 23 Gigabaud, resulting in the utilisation of $94 \%$ of the available spectrum.

We conducted two transmission experiments, sending data over $75 \mathrm{~km}$ of single-mode fibre in the laboratory as well as in a field trial across an installed metropolitan area single-mode fibre network (see Supplementary Note 1) connecting the Melbourne City campus of RMIT and the Clayton campus of Monash University, spanning the greater metropolitan area of Melbourne. Spectra of the comb at key points are given in Fig. 3a-c. At the receiver, the signal was recovered using a common offline digital signal processing (DSP) flow (see Methods). Figure 3d shows constellation diagrams for the signal at $194.34 \mathrm{THz}$. In the back-toback configuration (i.e., with the transmitter directly connected to the receiver) we measured a signal quality $\left(Q^{2}\right.$, from error-vector magnitude) approaching $18.5 \mathrm{~dB}$, dropping to near $17.5 \mathrm{~dB}$ when transmitting the fully modulated comb through the test links.

Transmission results. Figure 4a shows the transmission performance using the bit-error ratio (BER) for each channel as a metric. Three scenarios were investigated: (i) a direct connection between the transmitter stage to the receiver (back-to-back, B2B) and after transmission through (ii) in-lab fibre and (iii) over the field trial network. Transmission globally degraded the performance of all channels, as expected. As a performance benchmark, 

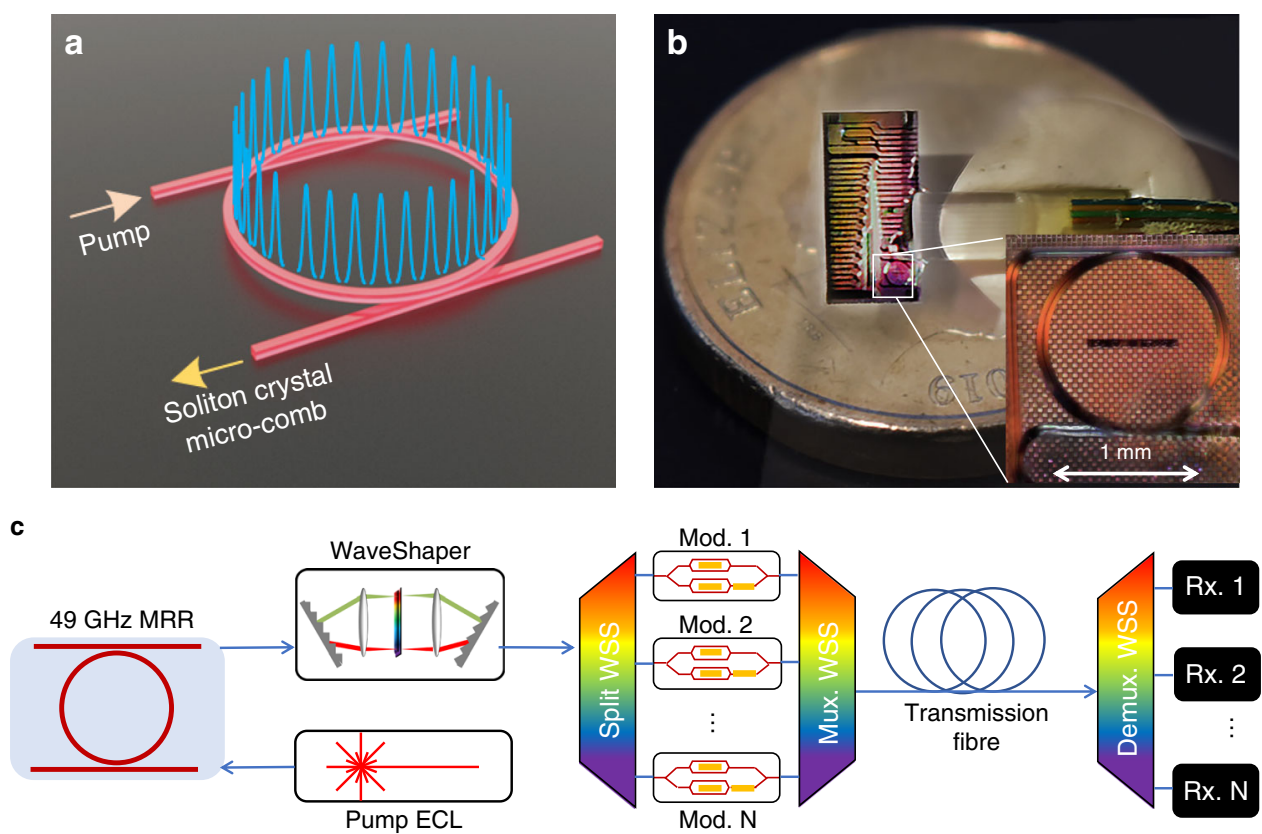

Fig. 1 Conceptual diagram of a soliton crystal micro-comb communications experiment. a. Illustration of the soliton crystal state used in this paper. We infer from the generated spectrum that the state was a single temporal defect crystal across the ring. The state had a characteristic 'scalloped' micro-comb spectrum, corresponding to the single temporal defect crystal state. $\mathbf{b}$ Photograph of the fibre-optic packaged micro-ring resonator chip used for soliton crystal generation. The full chip is $5 \mathrm{~mm} \times 9 \mathrm{~mm}$, of which we use devices and access waveguides on 1/4 of the area. The AUD $\$ 2$ coin (20.5 mm diameter) shown for scale is similar in size to a USD nickel or a 10 Euro cent coin. Inset is a microscope image of the ring resonator element, with a scale bar. Visible distortions are due to an overlayer of glue from the fibre array. c Experimental setup. A CW laser, amplified to 1.8 W, pumped a $48.9 \mathrm{GHz}$ FSR micro-ring resonator, producing a micro-comb from a soliton crystal oscillation state. The comb was flattened and optically demultiplexed to allow for modulation, and the resulting data optically multiplexed before the subsequent transmission through fibres with EDFA amplification. At the receiver, each channel was optically demultiplexed before reception. ECL edge-coupled laser, WSS wavelength-selective switch, $R x$ receiver.
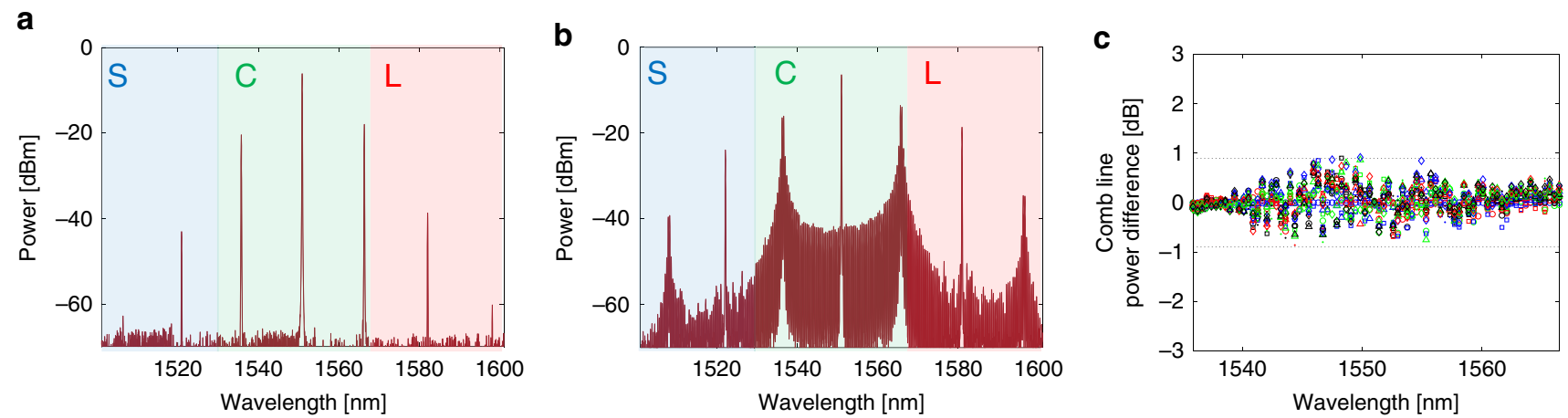

Fig. 2 Soliton crystal generation. To generate a soliton crystal, a laser is slowly tuned from the red side of a resonance to a pre-set wavelength. a A primary comb (Turing pattern) is initially generated as the laser is tuned into resonance with the ring. $\mathbf{b}$ Spectrum of the soliton crystal oscillation state used for experiments. The state had a characteristic 'scalloped' micro-comb spectrum, corresponding to the single temporal defect crystal state illustrated over the ring. At the pre-set wavelength, a soliton crystal forms, with spectral features based around the primary comb lines. The state that we use provides comb lines over most of the optical communications C-band. c Soliton crystal comb line power difference for 10 independent crystal generation instances (different symbols indicate distinct generation instances). Comb line powers remain within $\pm 0.9 \mathrm{~dB}$ of the initial spectrum, indicating reliable generation of the desired soliton crystal state.

Fig. 4a indicates a $20 \%$ soft-decision forward error correction (SD-FEC) threshold given at a BER of $4 \times 10^{-2}$ from a demonstrated $\operatorname{code}^{37}$. All results were below the given FEC limit, but since using SD-FEC thresholds based on BER is less accurate for higher-order modulation formats and for high BERs ${ }^{38}$, we additionally used generalised mutual information (GMI) to calculate the system performance. Figure $4 \mathrm{~b}$ plots the GMI for each channel and its associated SE, with lines given to indicate projected overheads. We achieved a raw bitrate (line-rate) of $44.2 \mathrm{~Tb} / \mathrm{s}$, which translates to an achievable coded rate of
$40.1 \mathrm{~Tb} / \mathrm{s}$ (in $\mathrm{B} 2 \mathrm{~B}$ ), dropping to $39.2 \mathrm{~Tb} / \mathrm{s}$ and $39.0 \mathrm{~Tb} / \mathrm{s}$ for the lab and field-trial transmission experiments, respectively. These yielded spectral efficiencies of $10.4,10.2$, and $10.1 \mathrm{~b} / \mathrm{s} / \mathrm{Hz}$ (see Methods). These data rates represent an increase of $\sim 50 \%$ (see Methods) over the highest reported result from a single integrated device ${ }^{28}$, whereas the spectral efficiency is 3.7 times higher. This is notable considering that the experiments were performed with the added penalty of full comb flattening (equalisation, even though this is in fact not necessary ${ }^{39}$ ), and without closed-loop feedback or stabilisation. 
a

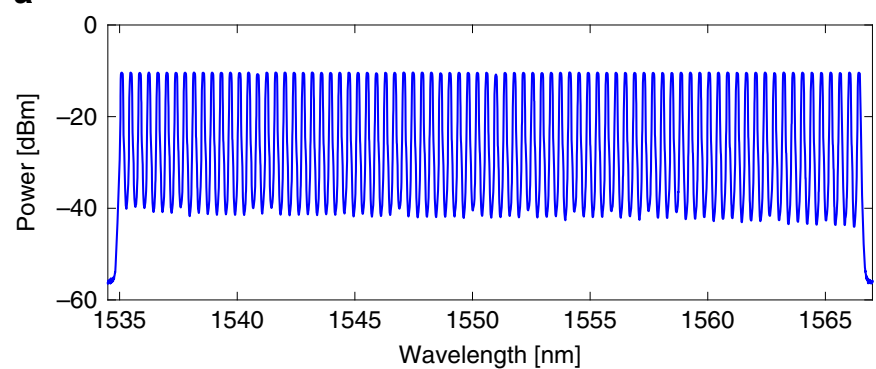

b

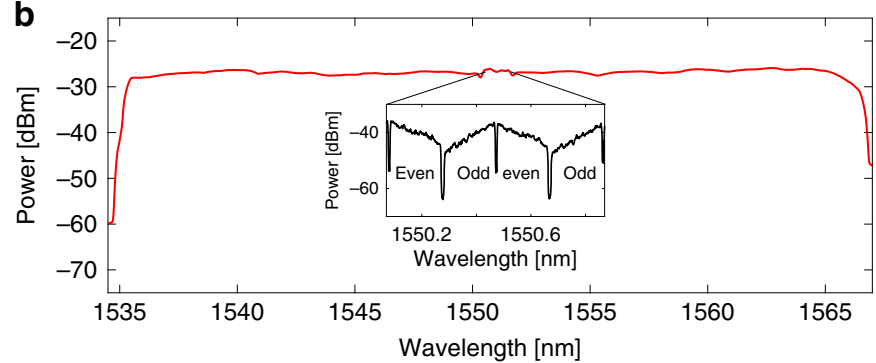

C

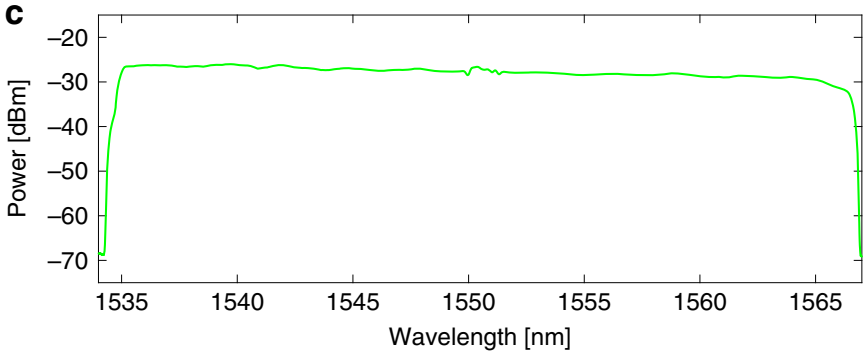

d

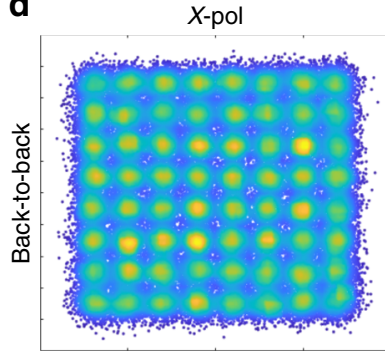

$Q^{2}[\mathrm{~dB}]: 18.4$, BER: $2.08 \times 10^{-2}$

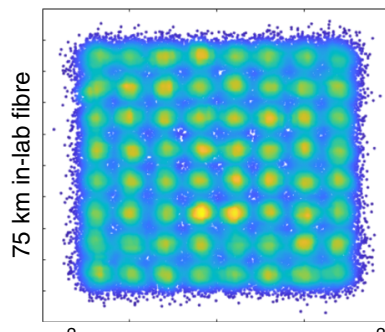

$Q^{2}[\mathrm{~dB}]: 18.1$, BER: $2.36 \times 10^{-2}$

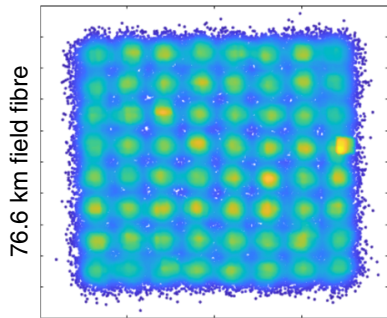

$Q^{2}[\mathrm{~dB}]: 17.5$, BER: $3.07 \times 10^{-2}$

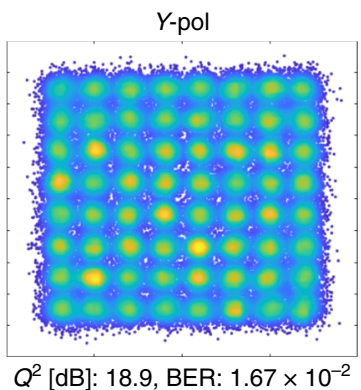

$Q^{2}[\mathrm{~dB}]: 18.9, \mathrm{BER}: 1.67 \times 10^{-2}$

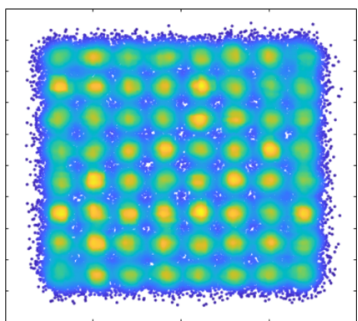

$Q^{2}[\mathrm{~dB}]: 18.5$, BER: $2.03 \times 10^{-2}$

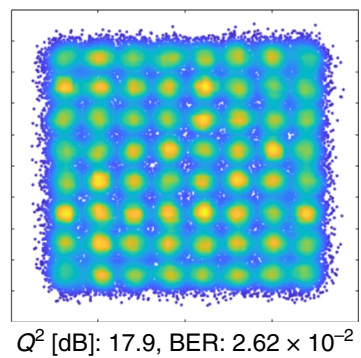

Fig. 3 Soliton crystal super-channel spectra, and indicative signal constellations. a-c Spectra of the soliton crystal frequency comb after flattening $\mathbf{a}$, modulation and transmission through either $75 \mathrm{~km}$ spooled in-lab fibre $\mathbf{b}$ or through the field-trial link $\mathbf{c}$. The spectrum $\mathbf{a}$ is measured with $12.5 \mathrm{GHz}$ resolution to resolve the individual comb lines, whereas $\mathbf{b}$ and $\mathbf{c}$ are plotted at $50 \mathrm{GHz}$ resolution to illustrate average channel powers. Flattening equalised the comb line power to within $1 \mathrm{~dB}$. After modulation and amplification, the channels were shaped by the EDFA gain spectrum. The inset in $\mathbf{b}$ depicts the test channel spectra captured with a $150 \mathrm{MHz}$ resolution optical spectrum analyser (Finisar WaveAnalyzer), highlighting the odd and even sub-bands modulated onto each comb line in the test band. $\mathbf{d}$ Constellation diagrams for a comb line at $193.4 \mathrm{THz}(1550.1 \mathrm{~nm})$ for both $\mathrm{X}$ - and $\mathrm{Y}$-polarisation channels. 'Back-to back' denotes the transmitter directly connected to the receiver, ' $75 \mathrm{~km}$ in-lab fibre' indicates reception after transmission through $75 \mathrm{~km}$ of spooled fibre inside the lab (as per $\mathbf{b}$ ), whereas '76.6 km field fibre' denotes reception after transmission through the field-trial link (as per c). BER and $\mathrm{Q}^{2}$ related to the constellations are noted on each.

\section{Discussion}

Table 1 summarises key results from the literature comparing the various system performance metrics for demonstrations based on a single integrated source and over standard fibre (or calculated on a per-mode basis for multicore fibre). Previous to this work, the best result (per core) ${ }^{28}$ was based on single micro-comb that was able to support $30.1 \mathrm{~Tb} / \mathrm{s}$ over the $\mathrm{C}$ and $\mathrm{L}$ bands, when using a standard tuneable laser coherent receiver. This is the benchmark result that we compare our results to since it is not only the best published result using a single micro-comb, but it closely resembles our experiment (single micro-comb at the transmitter, single tuneable laser at the receiver as a local oscillator). Note that our system uses less than half the spectrum of ref. ${ }^{28}$, whereas substantially exceeding its data rate, owing to our much higher spectral efficiency ( $3.7 \times$ higher). High modulation formats have also been achieved with dark solitons ${ }^{30}$, yet at a lower overall data rate, primarily owing to the high comb line spacing that significantly limits the spectral efficiency. The work of ref. ${ }^{4}$ used a comb generator based on a benchtop pulsed seed fibre laser source combined with waveguide spectral broadening. To provide a fully integrated system, this source would need to be on-chip. The focus in that experiment was using novel, proprietary multicore fibre to achieve a 30-fold increase in bandwidth over the fibre in this spatially multiplexed system, to reach 0.66 Petabits/s. On a per-mode basis ${ }^{4}$ this yields $25.6 \mathrm{~Tb} / \mathrm{s} /$ mode, a lower permode capacity than this work and that of ref. ${ }^{28}$. We note that both our approach and that of ref. ${ }^{28}$ are able to take advantage of SDM techniques to scale the overall bandwidth by using multicore fibre. We provide further comparisons in the Supplementary Note 3.

Our high transmission capacity and spectral efficiency are partly a result of the high conversion efficiency between the injected CW wave and the soliton crystal state 17,33 , which is reflected in the near absence of a soliton step (the jump in intracavity power that often occurs when generating coherent soliton states).

Although our experiments were restricted to the C-band, the soliton crystal comb (Fig. 2b) had a bandwidth exceeding $80 \mathrm{~nm}$. The comb lines in the $\mathrm{S}$ and $\mathrm{L}$ bands (1500-1535 $\mathrm{nm}$ and $1565-1605 \mathrm{~nm}$ ) could in principle be increased in power to enable transmission across all three bands, by varying the pump wavelength and power, tailoring dispersion, and/or by other methods. Assuming similar comb quality, this would result in a threefold increase in total data rate to $120 \mathrm{~Tb} / \mathrm{s}$ from a single integrated device. 

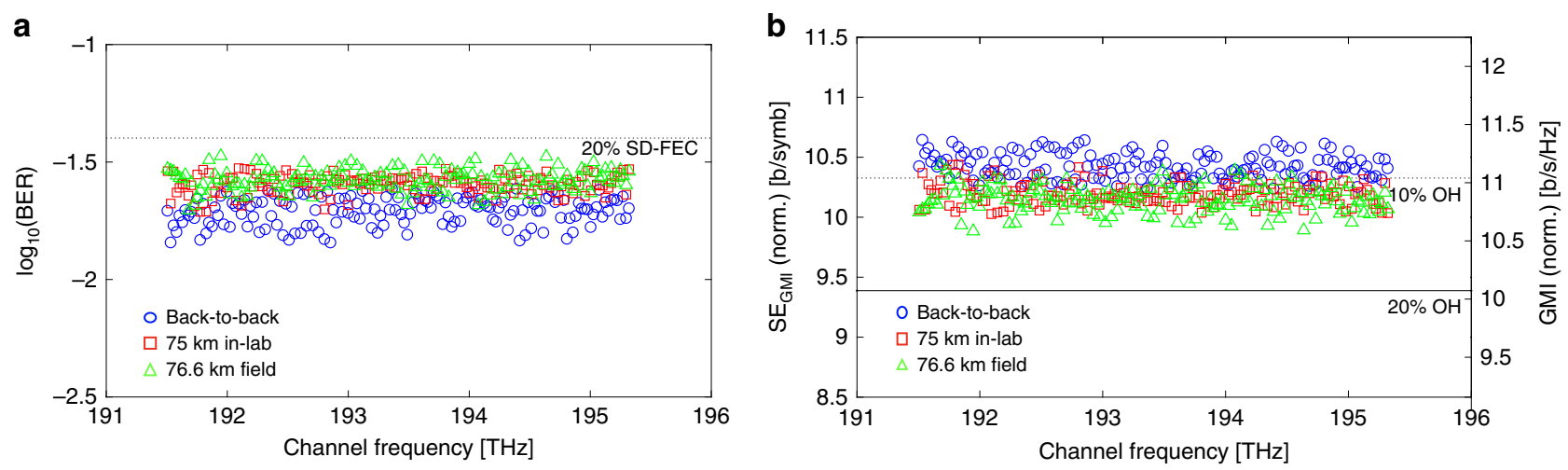

Fig. 4 Bit-error ratio, spectral efficiency and GMI for transmission experiment. a BER for each comb line. Blue circle points indicate the performance of the channels in a B2B configuration, red square dots are the performance after transmission through $75 \mathrm{~km}$ of in-lab spooled fibre, whereas the green triangles are after transmission through the $76.6 \mathrm{~km}$ installed metropolitan area fibre link. An indicative FEC threshold is given at $4 \times 10^{-2}$, corresponding to a pre-FEC error rate for a $20 \%$ soft-decision FEC based on spatially-coupled LDPC codes $^{2}$ (dashed line). After transmission, all channels were considered to be error-free. $\mathbf{b} \mathrm{GMI}$ and spectral efficiency measured for each comb line. GMI was calculated after normalisation to scale the measured constellations in order to account for the received signal-to-noise ratio (SNR). Lines are for $20 \%$ and $10 \%$ overheads. Spectral efficiency was derived from GMI, and the ratio of symbol rate to comb spacing. GMI indicates a higher overall capacity than BER with the indicated SD-FEC threshold, as GMI assumes the adoption of an ideal code for the system. For B2B, GMI (SE) varied between $11.3 \mathrm{~b} / \mathrm{symb}$. (10.6 b/s/Hz) and 10.9 b/symb. (10.3 b/s/Hz). After in-lab fibre transmission, the achievable per-channel GMI (SE) varied between $11.0 \mathrm{~b} / \mathrm{symb}$. $(10.4 \mathrm{~b} / \mathrm{s} / \mathrm{Hz})$ and $10.7 \mathrm{~b} / \mathrm{symb}$. $(10.1 \mathrm{~b} / \mathrm{s} / \mathrm{Hz})$, with the same range observed for the installed field-trial fibres. We estimate the overall capacity from the sum of the GMls, multiplied by the symbol rate.

Table 1 Key systems performance metrics.

\begin{tabular}{|c|c|c|c|c|c|}
\hline Line rate & Net rate & Format & Spectral efficiency & Transmission & Source \\
\hline $30.1 \mathrm{~Tb} / \mathrm{s}$ & $28.0 \mathrm{~Tb} / \mathrm{s}$ & 16 QAM & $2.8 \mathrm{~b} / \mathrm{s} / \mathrm{Hz}$ & 75 km SMF in-lab & Ref. 28 \\
\hline $4.8 \mathrm{~Tb} / \mathrm{s}^{\star}$ & $4.4 \mathrm{~Tb} / \mathrm{s}$ & 64 QAM & $1.1 \mathrm{~b} / \mathrm{s} / \mathrm{Hz}^{\star}$ & 80 km SMF in-lab & Ref. 30 \\
\hline $25.6 \mathrm{~Tb} / \mathrm{s}^{1}$ & $22.0 \mathrm{~Tb} / \mathrm{s}^{1}$ & 16 QAM & $3.2 \mathrm{~b} / \mathrm{s} / \mathrm{Hz}^{1}$ & 9.6 km, 30-core fibre ${ }^{1}$ & Ref. 41 \\
\hline $44.2 \mathrm{~Tb} / \mathrm{s}$ & $40.1 \mathrm{~Tb} / \mathrm{s}$ & 64 QAM & $10.4 \mathrm{~b} / \mathrm{s} / \mathrm{Hz}$ & B2B (0 km) & This work \\
\hline $44.2 \mathrm{~Tb} / \mathrm{s}$ & $39.2 \mathrm{~Tb} / \mathrm{s}$ & 64 QAM & $10.2 \mathrm{~b} / \mathrm{s} / \mathrm{Hz}$ & 75 km SMF in-lab & This work \\
\hline $44.2 \mathrm{~Tb} / \mathrm{s}$ & $39.0 \mathrm{~Tb} / \mathrm{s}$ & 64 QAM & $10.1 \mathrm{~b} / \mathrm{s} / \mathrm{Hz}$ & 76.6 km SMF installed & This work \\
\hline
\end{tabular}

Micro-combs with lower FSRs would support even higher spectral efficiencies since the signal quality improves at lower symbol rates. However, this may come at the expense of a narrower overall comb bandwidth. In our demonstration, singlesideband modulation enabled the multiplexing of two channels onto a single light source, effectively halving the comb spacing while improving back-to-back performance as limited by transceiver noise. This approach is made feasible by the stable nature of the soliton crystal combs. On the other hand, electro-optic modulation can also be used to sub-divide the repetition rate of micro-combs, which would enable broad comb-bandwidths. Although this would require locking the comb spacing to an external RF source, sub-MHz stabilisation of comb spacings has been reported ${ }^{40,41}$. Further, boosting the comb generation efficiency through laser cavity-soliton micro-combs ${ }^{42}$ may provide a powerful path to improve signal quality and system capacity even further. Finally, for newly deployed links, our approach can readily be combined with space division multiplexing using multicore fibre ${ }^{4,43}$, which would result in data rates of many petabit/s from a single source.

In conclusion, we report high-performance ultrahigh bandwidth optical transmission from a single integrated chip source by using soliton crystal micro-combs. This achievement is a result of the low comb spacing combined with the stable, efficient, and broad bandwidth of the soliton crystal combs, all enabled by their
CMOS-compatible integration platform. Soliton crystal microcombs are intrinsically coherent, low noise, and can be initialised and maintained using standard open-loop control with off-theshelf equipment. This work demonstrates their ability to support ultrahigh bandwidth data transmission in practical and demanding environments.

\section{Methods}

CMOS-compatible micro-comb source. The MRR for comb generation was fabricated using CMOS-compatible processes ${ }^{10,30,31}$ with doped silica glass waveguides, which features low linear loss $\left(\sim 0.06 \mathrm{~dB} \mathrm{~cm}^{-1}\right)$, a moderate nonlinear parameter $\left(\sim 233 \mathrm{~W}^{-1} \mathrm{~km}^{-1}\right)$, and negligible nonlinear loss that leads to an ultrahigh nonlinear figure of merit. The MRR has a cross-section of $3 \times 2 \mu \mathrm{m}$ and a radius of $\sim 592 \mu \mathrm{m}$, yielding an FSR of $48.9 \mathrm{GHz}$ and a Q factor $>1.5$ million. The dispersion of the TM mode was designed to be anomalous in the $\mathrm{C}$ band with a jump at $\sim 1552 \mathrm{~nm}$ brought about by the mode crossing. The bus waveguides of the MRR were directed to on-chip mode converters, then coupled to a single-mode fibre array, with a fibre-chip coupling loss of $\sim 0.5 \mathrm{~dB}$ per facet.

Although statistical studies of fabrication yield are outside the scope of this work, we note that our platform is fully CMOS-compatible, using stepper mask aligners on full wafers ${ }^{44}$. Further, our low index contrast (core index $=1.7$ ), results in larger waveguide dimensions, which in turn makes them less sensitive to fabrication error. Our typical yields for FSR and Q factor are extremely high-well above $90 \%$, and mode-crossings do not pose a particular challenge. We have fabricated many soliton crystal devices ${ }^{35}$ with high reproducibility. The discovery that mode-crossings provide a powerful approach towards achieving robust or even deterministic generation of micro-combs shows that further engineering of these structures remains an important and highly beneficial challenge that will yield new functionality. 
Soliton crystal micro-comb generation. The micro-comb used in the study was generated from the doped silica double-bus micro-ring resonator described above, packaged with a fibre array connection to all four device ports. We pumped the ring with a CW external cavity laser (Yenista Tunics-100S-HP) at an output power of $15 \mathrm{~mW}$, which was then amplified to $1.8 \mathrm{~W}$ in a polarisation maintaining erbium doped fibre amplifier (EDFA) (Pritel PMFA-37). Only the TM mode of the resonator oscillated in a soliton crystal state, hence the pump polarisation was tuned to match this mode. As indicated in Fig. 1a, we inserted the pump light into the 'through' port and collected light from the corresponding 'drop' port. The MRR chip was mounted on a Peltier cooler, monitored by a standard NTC temperature sensor. The temperature was maintained with a thermo-electric cooler (TCM-M207) at $25^{\circ} \mathrm{C}$, within $0.1^{\circ} \mathrm{C}$ of accuracy. The laser was set to standard running mode, with no extra steps made to stabilise the output frequency. Soliton crystal generation was achieved by automated wavelength tuning, in turn reducing the system complexity compared with other micro-comb generation schemes (see ref. ${ }^{12}$ and references within). We measured the internal conversion efficiency of our soliton crystals to be $42 \%$ for the whole spectrum, and $38 \%$ when selecting the 80 lines over the C-band, highlighting that over $90 \%$ of our available comb power is compatible with standard C-band equipment (see Supplementary Note 2).

The generated soliton crystal micro-comb was then flattened in two stages by two independent programmable optical filters (Finisar WaveShaper $4000 \mathrm{~S}$ ). The WaveShapers had an insertion loss of $5 \mathrm{~dB}$ each, in addition to any variable attenuation. The first had a static filter shape set to equalise each comb line to within $\sim 1 \mathrm{~dB}$ of each other, to coarsely match the generic shape of the soliton crystal state we chose to use. The second programmable filter was set each time that a new soliton crystal state was initiated, to equalise the comb line powers to within $<1 \mathrm{~dB}$ of each other, although we note that it was often unnecessary to change the filter profile when generating a new soliton crystal. Spectral shaping in a WDM transceiver using a comb source involved minimal extra complexity as only the addition of attenuators after the WDM demultiplexer was required to route each comb line to a separate modulator. The comb was then amplified by a further polarisation maintaining EDFA (Pritel PMFA-20-IO), before being divided for modulation. Prior to modulation, the optical signal-to-noise ratio (OSNR) of the individual comb lines was $>28 \mathrm{~dB}$ (see Supplementary Note 2).

The nonuniform spectrum of soliton crystal combs has been considered as a drawback, and so for this reason, as well as to facilitate easier comparison with prior work using micro-combs, we ensured that the optical frequency comb was flattened such that all lines were of equal power.

Comb flattening in fact is not necessary either in our experiments or other micro-comb demonstrations (e.g., ${ }^{28,30}$.), as all comb lines are typically wavelength demultiplexed into separate waveguides and sent to separate modulators. It is then straightforward to adjust the comb line power by variable attenuators, amplifiers, or even by varying the RF drive amplitude to the modulators. In fact, we expect better performance without comb flattening, as the higher power comb lines would need less attenuation and/or amplification before modulation, resulting in a higher OSNR, whereas the lower power comb lines would have essentially the same performance as reported here. Furthermore, using the raw spectrum would avoid the loss of the extra Waveshaper. Therefore, avoiding flattening (working with the raw spectrum) would in fact yield even higher system performance.

Systems experiment. The detailed experimental setup is shown in Supplementary Fig. 1. The transmitter used three separate complex Mach-Zehnder modulators to provide both odd and even test bands, as well as a loading band. The comb lines for each of these bands were split using another programmable filter (Finisar WaveShaper $4000 \mathrm{~S}$ ) and were then amplified before modulation. Three tones separated by $98 \mathrm{GHz}$ around the selected test frequency were directed to two separate modulators (Sumitomo Osaka Electric Company New Business T.SBXH1.5-20). The two modulators were driven at a symbol rate of 23 Gbaud, providing a per subband line rate (i.e., excluding overheads) of $23 \mathrm{Giga}$-symbols $/ \mathrm{s} \times 6 \mathrm{bits} / \mathrm{symbol} \times 2$ polarisations $=276 \mathrm{~Gb} / \mathrm{s}$. The sub-bands were shifted by $12 \mathrm{GHz}$ from the optical carrier, with one modulator providing a sideband down-shifted from the optical carrier, and the other an up-shifted sideband. This enabled higher fidelity modulation than simple $46 \mathrm{Gbd}$ single-carrier modulation, given the transceiver noise limitations we had in our system. The odd and even bands were de-correlated by adding a delay with an extra length of optical fibre in the 'even' path. A third modulator (Covega Mach-40 806) was used to modulate the loading bands, which consisted of a two Nyquist sub-carrier modulation scheme to mimic the structure of the odd and even test bands. The two bands were driven by pairs of the positive and negative differential outputs of the AWG (Keysight M8195A, $65 \mathrm{GSa} / \mathrm{s}$, $25 \mathrm{GHz}$ bandwidth), whereas the loading channels were driven by a separate independent output pair. The modulating waveforms were set to provide 64 QAM signals, pulse shaped by a $2.5 \%$ roll-off RRC filter, running at 23 Gigabaud. On a $48.9 \mathrm{GHz}$ grid, this provided a $94 \%$ spectral occupancy. The modulator optical outputs were each passed through a polarisation maintaining $3 \mathrm{~dB}$ power splitter, one output being delayed by a few metres of optical fibre and then rotated by $90^{\circ}$ using a polarisation beam splitter/combiner. This provided emulation of polarisation multiplexing by delay de-correlation. The odd, even and loading bands were all de-correlated from each other by means of different fibre delays of a few metres.
The odd and even channels were passively combined with a 3-dB power splitter, to maintain the pulse shape of the central channels. The combined test and loading bands were multiplexed by a further programmable filter (Finisar WaveShaper $4000 \mathrm{~S}$ ). The roll-off of the filters from this device did affect the outer channels of the test band and the neighbouring channels in the loading channels. After multiplexing the fully modulated comb was amplified to a set launch power. The Tx DSP is described in Supplementary Note 1.

The physical fibre-optic network geography is shown in Supplementary Fig. 2 and the schematic layout in Supplementary Fig. 1 (see Supplementary Note 1). The transmission link was comprised of two fibre cables connecting labs at RMIT University (Swanston St., Melbourne CBD) and Monash University (Wellington $\mathrm{Rd}$, Clayton). These cables were routed from the labs access panels, to an interconnection point with the AARNet's fibre network. The fibre links are a mix of OS1 and OS2 standard cables and include both subterranean and aerial paths. There is no active equipment on these lines, providing a direct dark fibre connection between the two labs. The total loss for these cables was measured to be $13.5 \mathrm{~dB}$ for the RMIT-Monash link and $14.8 \mathrm{~dB}$ for the Monash-RMIT paths. The cable lengths as measured by OTDR were both $38.3 \mathrm{~km}$ (totalling $76.6 \mathrm{~km}$ in loopback configuration). At Monash, an EDFA was remotely monitored and controlled using a $1310 \mathrm{~nm}$ fibre-ethernet connection running alongside the C-band test channels. The comb was amplified to $19 \mathrm{dBm}$ before launch, at Monash, and upon return to RMIT.

The receiver stage architecture is shown in Supplementary Fig. 1. Before photodetection, the signal was filtered by a programmable optical filter (Finisar WaveShaper $4000 \mathrm{~S}$ ) set to a $35 \mathrm{GHz}$ passband, in order to select the channel to be measured. The $35 \mathrm{GHz}$ passband was found to be an optimal setting in experiment (see Supplementary Note 1 for more detail). The output of the filter was amplified to $\sim 10 \mathrm{dBm}$ before being directed into a dual-polarisation coherent receiver (Finisar CPDV1200, $43 \mathrm{GHz}$ bandwidth). A local oscillator was provided by an Agilent N7714A laser tuned close to the comb line of interest, at $16 \mathrm{dBm}$ of output power. The photo-detected signals were digitised by the 80 -giga-samples-per second (GSa/s), 33-GHz bandwidth inputs of a Keysight oscilloscope (DSO-Z504A $50 \mathrm{GHz}, 160 \mathrm{GSa} / \mathrm{s}$ ). The digitised waveforms were forwarded to a PC for offline DSP. The DSP flow started with renormalisation, followed by overlap-add chromatic dispersion compensation, then a spectral peak search for frequency offset compensation, followed by frame synchronisation using a short BPSK header, before final equalisation. As the specific fibre types used along the link are not well known, the level of chromatic dispersion compensation was estimated through analysing the header correlation peak height. Equalisation occurred in two stages, with a training-aided least-means-squared equaliser performing preconvergence, the taps of which were sent to a blind multi-modulus equaliser. After equalisation, a maximum-likelihood phase estimator was used to mitigate phase noise, before the signal was analysed in terms of BER, error-vector magnitude (EVM) and GMI. Further details are included in Supplementary Note 1.

System performance metrics. After signal reconstruction using DSP, we measured the system performance based on three separate metrics: BER, EVM and GMI.

BER is measured by decoding a 1.1-Mbit-long random bit sequence that was grey-coded onto the 64-QAM constellation. As such, a BER of $9 \times 10^{-5}$ provides 100 errors.

Error-vector magnitude provides an alternative metric, which is directly related to the effective signal-to-noise ratio (SNR) measured at the receiver in the presence of uniform Gaussian noise. We use EVM to calculate signal quality factor $\left(Q^{2}[\mathrm{~dB}]\right)$ in Fig. 3 as $20 \log _{10}\left(1 / \mathrm{EVM}^{2}\right)$, with $\mathrm{EVM}=1 / \mathrm{SNR}^{0.5}$.

In systems employing higher-order modulation formats, GMI provides a more accurate measure of system performance than taking BER and assuming a certain SD-FEC threshold ${ }^{38}$. We use GMI to provide the key performance figures in this demonstration (i.e., net data rate and spectral efficiency). In this case, the achievable capacity (b/s) is calculated as the sum of individual channel GMIs (b/symbol) and multiplying by the symbol rate (symbols/s).

Spectral efficiency $(\mathrm{b} / \mathrm{s} / \mathrm{Hz})$ can also be calculated from GMI, by taking the mean of the channel GMIs (b/symbol), multiplying by the symbol rate (symbols/second) and dividing by the channel spacing $(\mathrm{Hz})$.

\section{Data availability}

The data that support the findings of this study are available from the corresponding authors upon reasonable request.

\section{Code availability}

The algorithms used for this study are standard, and are outlined in detail in the Supplementary Note 1. MATLAB scripts can be provided by the corresponding authors upon reasonable request.

Received: 1 February 2020; Accepted: 20 April 2020; Published online: 22 May 2020 


\section{References}

1. Cisco Visual Networking Index: Forecast and Methodology, 2016-2021 (Cisco, September 2017).

2. Winzer, P. J., Neilson, D. T. \& Chraplyvy, A. R. Fiber-optic transmission and networking: the previous 20 and the next 20 years. Opt. Express 26 24190-24239 (2018).

3. Winzer, P. J. \& Neilson, D. T. From scaling disparities to integrated parallelism: A decathlon for a decade. J. Lightwave Technol. 35, 1099-1115 (2017).

4. $\mathrm{Hu}, \mathrm{H}$. et al. Single-source chip-based frequency comb enabling extreme parallel data transmission. Nat. Photonics 12, 469-473 (2018).

5. Ataie, V. et al. Ultrahigh count coherent WDM channels transmission using optical parametric comb-based frequency synthesizer. J. Light. Technol. 33, 694-699 (2015).

6. Hillerkuss, D. et al. 26 Tbit s-1 line-rate super-channel transmission utilizing all-optical fast Fourier transform processing. Nat. Photonics 5, 364-371 (2011).

7. Del'Haye, P. et al. Optical frequency comb generation from a monolithic microresonator. Nature 450, 1214-1217 (2007).

8. Pasquazi, A. et al. Micro-combs: a novel generation of optical sources. Phys. Rep. 729, 1-81 (2018).

9. Gaeta, A. L., Lipson, M. \& Kippenberg, T. J. Photonic-chip-based frequency combs. Nat. Photonics 13, 158-169 (2019).

10. Kippenberg, T. J., Holzwarth, R. \& Diddams, S. A. Microresonator-based optical frequency combs. Science 332, 555-559 (2011).

11. Hänsch, T. W. Nobel lecture: passion for precision. Rev. Mod. Phys. 78, 1297-1309 (2006).

12. Hall, J. L. Nobel lecture: defining and measuring optical frequencies. Rev. Mod. Phys. 78, 1279-1295 (2006).

13. Haelterman, M., Trillo, S. \& Wabnitz, S. Dissipative modulation instability in a nonlinear dispersive ring cavity. Opt. Commun. 91, 401-407 (1992).

14. Leo, F. et al. Temporal cavity-solitons in one-dimensional Kerr media as bits in an all-optical buffer. Nat. Photonics 4, 471-476 (2010).

15. Herr, T. et al. Temporal solitons in optical microresonators. Nat. Photonics $\mathbf{8}$, 145-152 (2013).

16. Xue, X. et al. Mode-locked dark pulse Kerr combs in normal-dispersion microresonators. Nat. Photonics 9, 594-600 (2015).

17. Cole, D. C., Lamb, E. S., Del'Haye, P., Diddams, S. A. \& Papp, S. B. Soliton crystals in Kerr resonators. Nat. Photonics 11, 671-676 (2017).

18. Suh, M.-G., Yang, Q.-F., Yang, K. Y., Yi, X. \& Vahala, K. J. Microresonator soliton dual-comb spectroscopy. Science 354, 600-603 (2016).

19. Yu, M. et al. Silicon-chip-based mid-infrared dual-comb spectroscopy. Nat. Commun. 9, 1869 (2018)

20. Liang, W. et al. High spectral purity Kerr frequency comb radio frequency photonic oscillator. Nat. Commun. 6, 7957 (2015).

21. Spencer, D. T. et al. An optical-frequency synthesizer using integrated photonics. Nature 557, 81-85 (2018)

22. Trocha, P. et al. Ultrafast optical ranging using microresonator soliton frequency combs. Science 359, 887-891 (2018).

23. Suh, M.-S. \& Vahala, K. J. Soliton microcomb range measurement. Science 359, 884-887 (2018)

24. Kues, M. et al. On-chip generation of high-dimensional entangled quantum states and their coherent control. Nature 546, 622-626 (2017).

25. Reimer, C. et al. Generation of multiphoton entangled quantum states by means of integrated frequency combs. Science 351, 1176-1180 (2016).

26. Brasch, V. et al. Photonic chip-based optical frequency comb using soliton Cherenkov radiation. Science 351, 357-360 (2016).

27. Del'Haye, P. et al. Phase-coherent microwave-to-optical link with a selfreferenced microcomb. Nat. Photonics 10, 516-520 (2016).

28. Marin-Palomo, P. et al. Microresonator-based solitons for massively parallel coherent optical communications. Nature 546, 274-279 (2017).

29. Pfeifle, J. et al. Optimally coherent Kerr combs generated with crystalline whispering gallery mode resonators for ultrahigh capacity fiber communications. Phys. Rev. Lett. 114, 093902 (2015).

30. Fülöp, A. et al. High-order coherent communications using modelocked darkpulse Kerr combs from microresonators. Nat. Commun. 9, 1598 (2018).

31. Moss, D. J., Morandotti, R., Gaeta, A. L. \& Lipson, M. New CMOS-compatible platforms based on silicon nitride and Hydex for nonlinear optics. Nat. Photonics 7, 597-607 (2013).

32. $\mathrm{Xu}, \mathrm{X}$. et al. Photonic microwave true time delays for phased array antennas using a $49 \mathrm{GHz}$ FSR integrated optical micro-comb source. Photonics Res. J. 6, B30-B36 (2018).

33. $\mathrm{Xu}, \mathrm{X}$. et al. Advanced adaptive photonic RF filters based on an optical microcomb source with 80 taps. IEEE J. Lightwave Technol. 37, 1288-1295 (2019).

34. Lugiato, L. A., Prati, F. \& Brambilla, M. Nonlinear Optical Systems, (Cambridge University Press, 2015).

35. Wang, W. et al. Robust soliton crystals in a thermally controlled microresonator. Opt. Lett. 43, 2002-2005 (2018).

36. Bao, C. et al. Direct soliton generation in microresonators. Opt. Lett. 42 , 2519-2522 (2017).
37. K. Schuh, et al., Single Carrier 1.2 Tbit/s Transmission over $300 \mathrm{~km}$ with PM64 QAM at 100 GBaud, Proc. Optical Fiber Communications (OFC), Th5B.5 (San Diego, 2017)

38. Alvarado, A. et al. Replacing the soft-decision FEC limit paradigm in the design of optical communication systems. J. Lightwave Technol. 34, 707-721 (2016).

39. Torres-Company, V., et al., Laser frequency combs for coherent optical communications, J. Lightwave Technol. https://doi.org/10.1109/ JLT.2019.2894170 (2019).

40. Obrzud, E., Lecomte, S. \& Herr, T. Temporal solitons in microresonators driven by optical pulses. Nat. Photonics 11, 600-607 (2017).

41. Papp, S. B. et al. Microresonator frequency comb optical clock. Optica 1, 10-14 (2014).

42. Bao, H. et al. Laser cavity soliton micro-combs. Nat. Photonics. 13, 384-389 (2019).

43. Puttnam, B., et al., $2.15 \mathrm{~Pb} / \mathrm{s}$ Transmission Using a 22 Core Homogeneous Single-Mode Multi-Core Fiber and Wideband Optical Comb, Proc. European Conference on Optical Communications (ECOC), PDP 3.1, (Valencia, 2015).

44. Little, B. E. et al. Very high-order microring resonator filters for WDM applications. IEEE Photonics Technol. Lett. 16, 2263-2265 (2004).

\section{Acknowledgements}

We gratefully acknowledge support from Australia's Academic Research Network (AARNet-aarnet.edu.au) for supporting our access to the field-trial cabling through the project Australian Lightwave Infrastructure Research Testbed (ALIRT), and in particular Tim Rayner, John Nicholls, Anna Van, Jodie O’Donohoe and Stuart Robinson. A.M. and B.C. acknowledges funding through the Australian Research Council's Discovery and Linkage Infrastructure schemes (DP190102773, LE170100160). B.C. thanks Dr. Tobias Eriksson Japan, now at Infinera, Sweden for helpful advice regarding GMI calculations. R.M. acknowledges funding by the Natural Sciences and Engineering Research Council of Canada (NSERC) through the Strategic, Discovery, and Acceleration Grants Schemes, by the MESI PSR-SIIRI Initiative in Quebec, by the Canada Research Chair Program, as well as additional support by the Government of the Russian Federation through the ITMO Fellowship and Professorship Program (grant 074-U 01) and by the 1000 Talents Sichuan Program.

\section{Author contributions}

B.C., X.X. and D.J.M. developed the original concept. B.E.L. and S.T.C. designed and fabricated the integrated devices. B.C., M.T., A.B. and X.X. performed the experiments B.C., M.T., X.X., T.G.N., A.B., A.M. and D.J.M. all contributed to the development of the experiment. B.C., M.T., X.X., A.B., R.M., A.M. and D.J.M. contributed to the writing of the manuscript. B.C., J.W., A.M. and D.J.M. supervised the research.

\section{Competing interests}

The authors declare no competing interests, as defined by Springer Nature editorial policy (https://www.nature.com/authors/policies/competing.html)

\section{Additional information}

Supplementary information is available for this paper at https://doi.org/10.1038/s41467 020-16265-x.

Correspondence and requests for materials should be addressed to B.C. or D.J.M.

Peer review information Nature Communications thanks the anonymous reviewer(s) for their contribution to the peer review of this work.

Reprints and permission information is available at http://www.nature.com/reprints

Publisher's note Springer Nature remains neutral with regard to jurisdictional claims in published maps and institutional affiliations.

\section{(i)}

Open Access This article is licensed under a Creative Commons Attribution 4.0 International License, which permits use, sharing, adaptation, distribution and reproduction in any medium or format, as long as you give appropriate credit to the original author(s) and the source, provide a link to the Creative Commons license, and indicate if changes were made. The images or other third party material in this article are included in the article's Creative Commons license, unles indicated otherwise in a credit line to the material. If material is not included in the article's Creative Commons license and your intended use is not permitted by statutory regulation or exceeds the permitted use, you will need to obtain permission directly from the copyright holder. To view a copy of this license, visit http://creativecommons.org/ licenses/by/4.0/

(C) The Author(s) 2020 